\title{
Monitoring online media reports for the early detection of unknown diseases: insights from a retrospective study of COVID-19 emergence
}

\author{
Sarah Valentin ${ }^{1}$, Alizé Mercier ${ }^{2}$, Renaud Lancelot ${ }^{3}$, Mathieu Roche ${ }^{4}$, and Elena Arsevska ${ }^{2}$ \\ ${ }^{1}$ Cirad, UMR Land, Environment, Remote Sensing and Spatial Information (Tetis) \\ ${ }^{2}$ Cirad \\ ${ }^{3}$ CIRAD \\ ${ }^{4}$ Cirad, UMR Land, Environment, Remote Sensing and Spatial Information (Tetis)
}

April 28, 2020

\begin{abstract}
Event-based surveillance (EBS) systems monitor a broad range of information sources to detect early signals of disease emergence, including new and unknown diseases. Following the emergence of a newly identified coronavirus -so-called COVID-19, in humans in December 2019 in Wuhan, China, we conducted a retrospective analysis of the capacity of three Event-Based Systems (EBS) systems (ProMED, HealthMap and PADI-web) to detect early signals of this emergence. We evaluated the changes in the online news vocabulary coinciding with the period before / after the identification of COVID-19, as well as the assessment of its contagiousness and pandemic potential. ProMED was the timeliest EBS, detecting signals one day before the official notification. At this early stage, the specific vocabulary was related to "pneumonia symptoms" and "mystery illness". Once COVID-19 was identified, the vocabulary changed to virus family and specific COVID-19 acronyms. Our results suggest the three EBS systems are complementary regarding data sources, and all need improvements regarding timeliness. EBS methods should be adapted to the different stages of disease emergence to improve the early detection of future emergence of unknown pathogens.
\end{abstract}

\section{Title:}

Running head: Detection of COVID-19 emergence in online news.

Sarah Valentin ${ }^{1,2}$, Alizé Mercier ${ }^{2}$, Renaud Lancelot ${ }^{2}$, Mathieu Roche $^{1}$ and Elena Arsevska ${ }^{*}+$ ${ }^{1}$ UMR TETIS, CIRAD, F-34398 Montpellier, France. TETIS, Univ Montpellier, AgroParisTech, CIRAD, CNRS, INRAE, Montpellier, France. ${ }^{2}$ UMR ASTRE, CIRAD, F-34398 Montpellier, France. ASTRE, Univ Montpellier, CIRAD, INRAE, Montpellier, France. ${ }^{*}$ Corresponding author. ${ }^{+}$E-mail: elena.arsevska@cirad.frArticle type: Rapid communicationWords counts: 2,115 wordsAbstract Event-based surveillance (EBS) systems monitor a broad range of information sources to detect early signals of disease emergence, including new and unknown diseases. Following the emergence of a newly identified coronavirus -so-called COVID-19, in humans in December 2019 in Wuhan, China, we conducted a retrospective analysis of the capacity of three Event-Based Systems (EBS) systems (ProMED, HealthMap and PADI-web) to detect early signals of this emergence. We evaluated the changes in the online news vocabulary coinciding with the period before / after the identification of COVID-19, as well as the assessment of its contagiousness and pandemic potential. ProMED was the timeliest EBS, detecting signals one day before the official notification. At this early stage, the specific vocabulary was related to "pneumonia symptoms" and "mystery illness". Once COVID-19 was identified, the vocabulary changed to virus family and specific COVID-19 acronyms. Our results suggest 
the three EBS systems are complementary regarding data sources, and all need improvements regarding timeliness. EBS methods should be adapted to the different stages of disease emergence to improve the early detection of future emergence of unknown pathogens.Keywords: epidemic intelligence, online news, emerging disease, PADI-web, COVID-19, One Health

\section{Introduction}

Epidemic intelligence (EI) aims to detect, monitor and assess potential health threats for early warning and rapid response (Paquet et al., 2006). In addition to the indicator-based surveillance of official sources, public and animal health agencies increasingly integrate an event-based surveillance (EBS) component to their EI system. The EBS uses unstructured data from unofficial sources such as online news to improve the early detection of emerging infectious diseases (EIDs). Since the late 1990s, several free-access EBS systems support the EI process, such as the Program for Monitoring Emerging Disease or ProMED (Woodall, 2001), HealthMap (Brownstein et al., 2008) and since recently PADI-web (Valentin, Arsevska, et al., 2020). ProMED is a human-curated system launched in 1994 by the International Society for Infectious Diseases (ISID). The system relies on a large network of experts worldwide who produce and share verified reports on disease outbreaks in a common platform (Carrion \& Madoff, 2017). HealthMap is a semi-automated system founded by the Boston Children's Hospital in 2006. The tool monitors both official and non-official news sources on the web (Freifeld et al., 2008). Both HealthMap and ProMED monitor a broad range of human, animal and environmental known and unknown threats. The Platform for Automated extraction of animal Disease Information from the web (PADI-web) was created in 2016 to monitor online animal health-related news for the French Epidemic Intelligence System (FEIS) (Arsevska et al., 2018, Valentin et al., 2020). Both HealthMap and PADI-web automatically retrieve health-related news from Google News using customized Really Simple Syndication (RSS) feeds. For the detection of news, the two systems use terms for known diseases and terms for clinical signs and syndromes (Arsevska et al., 2016). All three EBS systems monitor news in multiple languages, including Chinese.

On Dec. 31 2019, local health officials of the Chinese city of Wuhan reported a cluster of 27 cases of "pneumonia of unknown cause". These cases were linked to a wholesale seafood market in the city. In January 2020, the first death was reported, and the aetiology was identified as a new coronavirus, SARS-Cov-2, and the disease was named COVID-19. The first epidemiological study on patients with laboratory-confirmed COVID-19 infection reported an onset of illness as early as Dec. 12019 (Huang et al., 2020).

This retrospective study aimed first to evaluate three EBS systems (ProMED, HealthMap and PADI-web) and their capacity to detect the COVID-19 emergence in China timely. Secondly, we focused on PADI-web to understand how an animal health EBS system contributed to the detection of a human EID. We analysed the RSS feeds from PADI-web that detected the COVID-19-related news articles (further referred to as "news"). Thirdly, we assessed the vocabulary in the news detected by PADI-web and its change in relation to the identification of the pathogen and the spread of the EID.

\section{Material and methods}

\subsection{Detection of COVID-19-related news}

To assess the timeliness of the three EBS, we searched for news from Dec. $1^{\text {st }}$, to Dec. 31, 2019. We compared the first news regarding publication date, language, and source.

To understand how PADI-web detected the COVID-19 emergence, we further filtered a second corpus of news published from Dec. 31, 2019, to Jan. 26, 2020, containing at least one of the following words in the title and body of the news: "pneumonia", "respiratory illness", "coronavirus", "nCoV" (an early name for COVID-19), and "Wuhan". After manual verification of their relevance, we retained for analysis 275 out of 333 news (Valentin, Mercier, et al., 2020).

To assess the link between the animal health RSS feeds from PADI-web and the detected news, we analysed them according to the RSS feed used for their retrieval. To this end, we read each news and categorized it 
into i) disease-specific RSS feeds (containing specific disease names), and ii) syndromic RSS feeds (containing combinations of symptoms and animal hosts).

\subsection{Vocabulary in the news}

To analyse the vocabulary change through the period ranging from COVID-19 discovery to its spread outside China, we extracted terms from the whole corpus. For this purpose, we used a ranking function based on terms frequency and importance11Using the F-TFIDF-C measure (Lossio-Ventura et al., 2014) with the support of BioTex, a text-mining tool adapted to the biomedical area (Lossio-Ventura et al., 2016). BioTex is based on the use of (i) a relevant combination of information retrieval techniques and statistical methods, and (ii) a list of syntactic structures of the terms that have been learnt with relevant sources (e.g. MeSH). The terms extracted with BioTex can be simple (e.g. influenza), or compound (e.g. avian influenza), and are lowercased. We further identified the terms referring to COVID-19, such as "new virus" and "mystery pneumonia". We manually categorized the terms as "mystery" (terms referring to the unknown threat), "pneumonia" (terms referring to the clinical signs), "coronavirus" (terms referring to the virus taxonomy) and "technical" (technical acronyms for the virus itself). One news can contain terms from different categories. We calculated the daily proportion of each category, expressed as the sum of the occurrences of the category divided by the total number of occurrences.

\section{Results}

3.1. Detection of newsProMED was the first to detect and report a news from a Chinese online source22https://promedmail.org/promed-post/?id=6864153\}. The ProMED report dated from Dec. 30, 2019, one day before the first official notification of pneumonia-like cases in Wuhan (Wuhan Municipal Health Commission, 2020). PADI-web and HealthMap respectively detected three and one COVID-19related news on Dec. 31, 2019, the same day as the first official notification of pneumonia-like cases in Wuhan (one HealthMap news from an English source, three PADI-web news from two English and one Chinese source). The news detected by the three EBS originated from five different media sources. From 275 COVID-19-related news retrieved by PADI-web, $45.5 \%(n=125)$ were retrieved by disease-specific RSS, and the remaining $54.5 \%$ ( $n=150$ ) were retrieved by syndromic RSS feeds (Table 1). Content-wise, $31.7 \%$ $(n=87)$ of the news compared COVID-19 to five animal diseases (avian influenza, African swine fever, classical swine fever, West Nile virus, and Rift Valley fever), $24.4 \%(n=67)$ of the news described the broad range of animal species susceptible to coronaviruses, $18.2 \%(n=50)$ described ruling-out avian influenza from diagnosis of COVID-19, and 7.7\% $(n=21)$ described ongoing outbreaks in addition to COVID-19 (avian influenza, African swine fever, classical swine fever, and foot-and-mouth disease), $2.5 \%(n=7)$ referred to animal species present in the Chinese markets as being the potential COVID-19 source, and 0.7\% $(n=2)$ news advised to avoid contact with animals. Irrelevant keywords matches were found in 12 news (e.g. finding a host keyword in the name of a source), and no link could be established between the RSS feed and the article for 29 remaining news (10.5\%).

\subsection{Vocabulary of the news}

From the terms referring either to the virus or the disease, 18 terms belonged to the category "pneumonia", 8 terms belonged to the category "mystery", 3 terms belonged to the category "coronavirus" (one of them, "coronovirus" being a misspelt form for "coronavirus"), and 7 terms belonged to the category "technical" (Table 2 ).

Before identification of the virus (Dec. 31, 2019 - Jan. 8, 2020), 58.1\% $(n=317 / 586)$ of the COVID-19 terms were from category "pneumonia", $29.1 \%(n=159 / 586)$ were from category "mystery" and $12.8 \%(n=70 / 586)$ were from category "coronavirus". From the official identification to the first report of a case outside China (Jan. 09, 2020 - Jan. 12, 2020), 48.5\% ( $n=127 / 262)$ of the terms were from category "coronavirus", $34.7 \%$ ( $n=91 / 262)$ were from category "pneumonia", and $16.8 \%(n=44 / 262)$ were from category "mystery". From this first report to the confirmation of human-to-human transmission (Jan. 13, 2020 - Jan. 19, 2020), $58.3 \%$ ( $n=196 / 336)$ of the terms were from category "coronavirus", $27.4 \%(n=92 / 336)$ were from category "pneumonia", $11.3 \%$ ( $n=38 / 336)$ were from category "mystery", and 3.0\% $(n=10 / 336)$ were from category 
"technical". From the confirmation of human-to-human transmission to the end of the studied period $62.9 \%$ ( $n=906 / 1440)$ of the terms were from category "coronavirus", $17.4 \%(n=250 / 1440)$ were from category "technical", $14.1 \%(n=203 / 1140)$ were from category "pneumonia", and 5.6\% ( $n=81 / 1140)$ were from category "mystery" (Figure 1 ).

Among the three compared EBS systems, only ProMED relies on local expert information to alert on health threats. This result suggests the network of local experts rooted in the field is crucial for the detection of EID events and their reporting. On the other hand, HealthMap and PADI-web detected the news on the same day than the official reporting. Thus, it is important to understand their current limitations and stress the role of experts in EBS systems. Further studies should also assess whether the timeliness of automated systems depends on the communication strategies of online media, what their threshold for reporting health events is, and how these features influence the sensitivity of web-based EBS systems.

The three EBS systems included in this study monitor media in multiple languages, thus making it possible to detect news from local media. A further increase in the number of available languages should improve the sensitivity of the EBS systems (Barboza et al., 2014). Our study also showed that the three EBS systems are complementary regarding scope (animal health, animal and public health), moderation (manual, semiautomated, automated), and the number of covered languages.

PADI-web could retrieve news through animal-health related RSS feeds, thus proving useful to detect relevant information for public health risk assessors. For example, many of the news detected by PADI-web compared the magnitude and the economic impact of COVID-19 with avian influenza and African swine fever outbreaks in China. Indeed, before the identification of COVID-19, the pneumonia-like illness was compared to avian influenza zoonotic infections. Some news also presented a summary of several recent disease outbreaks in China, including African swine fever (which is not a zoonotic disease), thus explaining why they were captured.

The ability of EBS tools to embrace a broad scope of health-related topics through a limited number of queries (RSS feeds) is a significant strength compared to formal sources. This capacity largely depends on the intrinsic features of online news in which outbreak-related content is often enriched with additional information, such as comparisons with previous disease outbreaks, thus increasing the probability of being detected by EBS tools. However, the probability of detection of an EID event might be higher for (actually or assumed) zoonotic diseases and countries with ongoing animal disease outbreaks. This is not a strong limitation in practice.

In addition to syndromic feeds, disease-specific feeds equally contributed to the first detection of COVID-19 by PADI-web, thus highlighting the importance of combining both disease-specific and syndromic feeds. Furthermore, the vocabulary used to describe COVID-19 emergence before the virus identification included terms semantically related to "unknown" and "mysterious" events. Therefore, their integration into existing RSS feeds may increase the detection and retrieval of relevant news. We suggest enriching the identification of classic epidemiological entities (e.g. disease, hosts, locations, dates) in the news content with this category of terms.

Our results indicated that the vocabulary changes as the disease spreads. Thus, EBS methods used to retrieve and analyse news from the web should be adapted to the different stages of disease epidemiology. With MERS-CoV in 2014 and SARS in 2003, COVID-19 is the third coronavirus emergence in the past two decades, highlighting the need to monitor closely the emergence of pneumonia-like illnesses using existing EBS systems. Our results highlight the complementarity of existing systems and underline the need for collaborative development. Mutualizing resources from veterinary and public health seems crucial to improve the early detection of unknown diseases in a One Health context. Future work will focus on identifying the most relevant keywords for the rapid detection of unknown threats, in collaboration with other EBS systems. Also, EBS tools may be used in a broader perspective, such as monitoring the implementation of protective and control measures. These efforts invested in improving the timeliness and sensitivity of EBS systems only make sense if their output (alerts of EID events) is designed, supervised, and interpreted by epidemiologists 
in collaboration with disease experts and reference laboratories. Most importantly, this output should feed the information flow used by the health managers and decision-makers, for an early reaction to control EID events before they can spread.Acknowledgements The authors acknowledge ProMED for data sharing. This work was in part funded by the H2020 "Monitoring outbreak events for disease surveillance in a data science context" (MOOD) project under grant agreement No 874850 (https://mood-h2020.eu/)., the French General Directorate for Food (DGAL), the French Agricultural Research Centre for International Development (CIRAD) and the SONGES Project FEDER and Occitanie. This work was supported by the French National Research Agency under the Investments for the Future Program, referred to as ANR-16CONV-0004.Data Sharing and AccessibilityThe data that support the findings of this study are openly available in CIRAD Dataverse at http://doi.org/doi:10.18167/DVN1/MSLEFC.Conflict of interests

The authors declare no conflict of interests.

\section{Ethics Statement}

The authors confirm that the ethical policies of the journal, as noted on the journal's author guidelines page, have been adhered to. No ethical approval was required because this study did not involve any experimental protocol on humans or animals, and only uses publicly available online data.

\section{References}

Arsevska, E., Roche, M., Hendrikx, P., Chavernac, D., Falala, S., Lancelot, R., \& Dufour, B. (2016). Identification of terms for detecting early signals of emerging infectious disease outbreaks on the web. Computers and Electronics in Agriculture , 123 , 104-115. https://doi.org/10.1016/j.compag.2016.02.010

Arsevska, E., Valentin, S., Rabatel, J., de Goer de Herve, J., Falala, S., Lancelot, R., \& Roche, M. (2018). Web monitoring of emerging animal infectious diseases integrated in the French Animal Health Epidemic Intelligence System. PLOS ONE , 13 (8), e0199960. https://doi.org/10.1371/journal.pone.0199960

Barboza, P., Vaillant, L., Le Strat, Y., Hartley, D. M., Nelson, N. P., Mawudeku, A., Madoff, L. C., Linge, J. P., Collier, N., Brownstein, J. S., \& Astagneau, P. (2014). Factors influencing performance of internet-based biosurveillance systems used in epidemic intelligence for early detection of infectious diseases outbreaks. PLoS ONE ,9 (3), e90536. https://doi.org/10.1371/journal.pone.0090536

Brownstein, J. S., Freifeld, C. C., Reis, B. Y., \& Mandl, K. D. (2008). Surveillance Sans Frontieres: Internetbased emerging infectious disease intelligence and the HealthMap project. PLoS Medicine ,5 (7), e151.

Carrion, M., \& Madoff, L. C. (2017). ProMED-mail: 22 years of digital surveillance of emerging infectious diseases. International Health , 9 (3), 177-183. https://doi.org/10.1093/inthealth/ihx014

Freifeld, C. C., Mandl, K. D., Reis, B. Y., \& Brownstein, J. S. (2008). HealthMap: Global Infectious Disease Monitoring through Automated Classification and Visualization of Internet Media Reports. Journal of the American Medical Informatics Association ,15 (2), 150-157. https://doi.org/10.1197/jamia.M2544

Huang, C., Wang, Y., Li, X., Ren, L., Zhao, J., Hu, Y., Zhang, L., Fan, G., Xu, J., Gu, X., Cheng, Z., Yu, T., Xia, J., Wei, Y., Wu, W., Xie, X., Yin, W., Li, H., Liu, M., .. Cao, B. (2020). Clinical features of patients infected with 2019 novel coronavirus in Wuhan, China.The Lancet , 395 (10223), 497-506. https://doi.org/10.1016/S0140-6736(20)30183-5

Lossio-Ventura, J. A., Jonquet, C., Roche, M., \& Teisseire, M. (2014). Towards a Mixed Approach to Extract Biomedical Terms from Text Corpus:International Journal of Knowledge Discovery in Bioinformatics ,4 (1), 1-15. https://doi.org/10.4018/ijkdb.2014010101

Lossio-Ventura, J. A., Jonquet, C., Roche, M., \& Teisseire, M. (2016). Biomedical term extraction: Overview and a new methodology.Information Retrieval Journal , 19 (1), 59-99. https://doi.org/10.1007/s10791-015$9262-2$ 
Paquet, C., Coulombier, D., Kaiser, R., \& Ciotti, M. (2006). Epidemic intelligence: A new framework for strengthening disease surveillance in Europe. Eurosurveillance, 11 (12), 5-6. https://doi.org/10.2807/esm.11.12.00665en

Valentin, S., Arsevska, E., Falala, S., de Goer, J., Lancelot, R., Mercier, A., Rabatel, J., \& Roche, M. (2020). PADI-web: A multilingual event-based surveillance system for monitoring animal infectious diseases. Computers and Electronics in Agriculture , 169 , 105163. https://doi.org/10.1016/j.compag.2019.105163

Valentin, S., Mercier, A., Lancelot, R., Roche, M., \& Arsevska, E. (2020). PADI-web COVID-2019 corpus: News articles manually labelled [Data set]. CIRAD Dataverse. https://doi.org/10.18167/DVN1/MSLEFC

Woodall, J. P. (2001). Global surveillance of emerging diseases: The ProMED-mail perspective. Cadernos de Saude Publica, 17, S147-S154.

Wuhan Municipal Health Commision. (2020, December 31). Information session of the Wuhan Municipal Health and Health Commission on the current situation of the pneumonia epidemic in our city.http://wjw.wuhan.gov.cn/front [in Chinese]

\begin{tabular}{llll}
\hline Link with COVID-2019 & Type of RSS feed & Type of RSS feed & Type of RSS feed \\
\hline & Disease-specific & Syndromic & Total \\
Comparison with another disease & $20.4 \%(n=56)$ & $11.3 \%(n=31)$ & $31.7 \%(n=87)$ \\
Disease ruled out & $17.8 \%(n=49)$ & $0.4 \%(n=1)$ & $18.2 \%(n=50)$ \\
Aggregation with other disease outbreaks & $6.2 \%(n=17)$ & $1.5 \%(n=4)$ & $7.7 \%(n=21)$ \\
Coronaviruses in animals & - & $24.4 \%(n=67)$ & $24.4 \%(n=67)$ \\
Market animals & - & $2.5 \%(n=7)$ & $2.5 \%(n=7)$ \\
Avoid contact with animals & - & $0.7 \%(n=2)$ & $0.7 \%(n=2)$ \\
Irrelevant keywords matches & $0.4 \%(n=1)$ & $4.0 \%(n=11)$ & $4.4 \%(n=12)$ \\
Unknown & $0.7 \%(n=2)$ & $9.8 \%(n=27)$ & $10.5 \%(n=29)$ \\
Total & $\mathbf{4 5 . 5 \% ( n = 1 2 5 )}$ & $\mathbf{5 4 . 5 \% ( n = 1 5 0 )}$ & $\mathbf{1 0 0 \% ( 2 7 5 )}$ \\
\hline
\end{tabular}

Table 1. Percentage (\%) and number (n) of COVID-19-related news retrieved by PADI-web from $31^{\text {st }}$ Dec. 2019 to $26^{\text {th }}$ Jan. 2020. Each article is categorized by type of feed (disease-related or syndromic) according to the link between the feed and COVID-19.

\begin{tabular}{ll}
\hline Category & Terms \\
\hline pneumonia & pneumonia, respiratory outbreak, lung disease, respiratory tract illness, respiratory illness, respiratory infecti \\
mystery & mystery, mysterious, unidentified, undocumented, disease x, unknown, abnormal, unexplained \\
technical & 2019-ncov, ncov, 2019 novel coronavirus, n-cov2019, novel coronavirus 2019, ncov2019, cov2019 \\
coronavirus & coronavirus, betacoronavirus, coronovirus \\
\hline
\end{tabular}

Table 2. Terms, used to describe SARS-CoV-2 and COVID-19 in the corpus and their corresponding category after manual classification. 


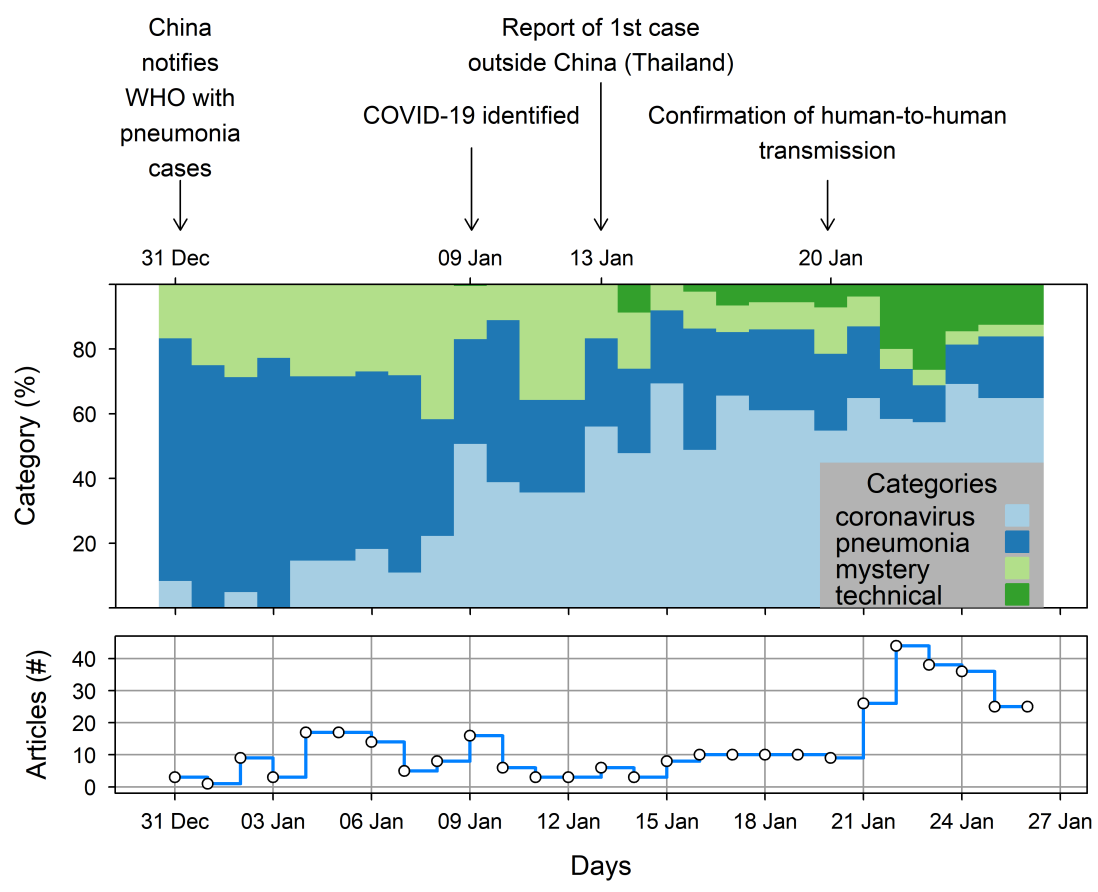

